factor therapy are scarce, especially in Asian populations. We assessed the effectiveness of infliximab (IFX) and azathioprine on PFCD and explored predictors of 'deep remission' based on clinical and radiologic assessments.

Methods Patients with Crohn's disease and active anal fistulas attending our centre for IFX therapy were prospectively enrolled. Each patient underwent clinical examination according to the Fistula Drainage Assessment Index, magnetic resonance imaging (MRI) to determine Van Assche score, $\mathrm{Ng}$ score, and main fistula length, endoscopy, assessment of Crohn's disease activity index (CDAI) and perianal Crohn's disease activity index (PCDAI), and laboratory tests up to 2 weeks prior to the start of and up to 2 weeks after the sixth IFX therapy (Week 32). Patients with PFCD treated with other medicines such as FK 506 or mesalazine and evaluated by two MRIs were retrospectively enrolled.

Results Of 38 patients treated with IFX, 52.6\% achieved clinical remission, and $42.1 \%$ achieved deep remission. The only predictor of deep remission was simple fistula $(\mathrm{p}=0.004$, odds ratio $=3.802,95 \%$ confidence interval: 1.541-9.383). Van Assche score (from $14.5 \pm 4.26$ to $7.36 \pm 7.53$; figure 1 ), CDAI (from $170 \pm 92$ to $71 \pm 69$ ), and PCDAI (from $7.45 \pm 2.65$ to $2.44 \pm 3.2$ ) decreased significantly after six IFX treatments. Our findings suggest that Van Assche score has some limitations related to its inability to reflect new fistulas and abscesses in some circumstances. We used $\mathrm{Ng}$ score to compare the changes between the initial and follow-up MRIs in each patient, which is more objective and suitable for monitoring the therapeutic effect.

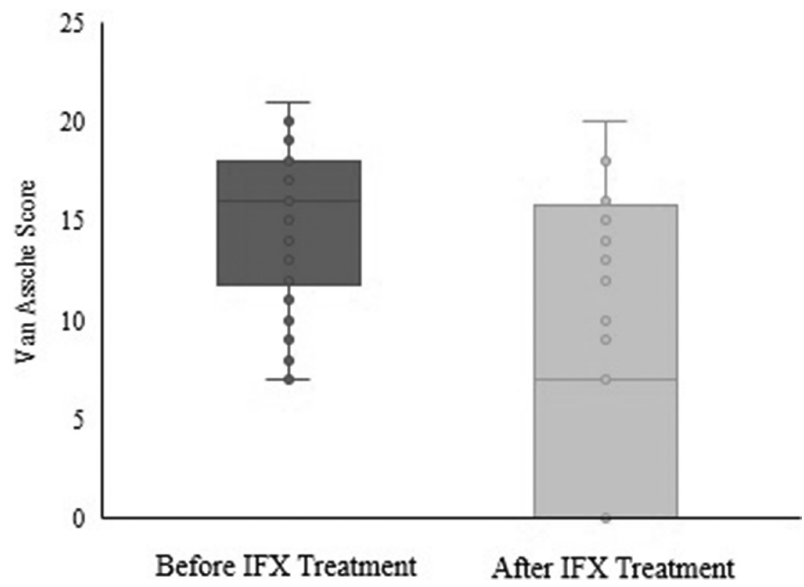

Abstract IDDF2018-ABS-0222 Figure 1 Van assche scores were significantly reduced after six infliximab treatments
Conclusions IFX is effective for the treatment of PFCD. MRI is the gold standard for evaluating PFCD, but Van Assche score has some limitations.

\section{IDDF2018-ABS-0223 AN EASTERN CHINESE MULTICENTER SURVEY ON HEALTH-RELATED QUALITY OF LIFE IN INFLAMMATORY BOWEL DISEASE PATIENTS AND DISEASE-RELATED PERCEPTION OF CAREGIVERS}

${ }^{1}$ Chenwen Cai*, ${ }^{2}$ Naizhong Hu, ${ }^{3}$ Yihong Fan, ${ }^{4}$ Chengdang Wang, ${ }^{5}$ Chunxiao Chen, ${ }^{1}$ Jun Shen, ${ }^{1}$ Meilan Huang, ${ }^{1}$ Di Zhao, 'Lijie Lai, ${ }^{1}$ Tianrong Wang, ${ }^{1}$ Yuqi Qiao, ${ }^{1}$ Qing Zheng, 'Zhihua Ran. 'Division of Gastroenterology and Hepatology, Renji Hospital, School of Medicine, Shanghai Jiao Tong University; Shanghai Inflammatory Bowel Disease Research Center, Shanghai, China; ${ }^{2}$ Department of Gastroenterology, The First Affiliated Hospital of Anhui Medical University, Hefei, Anhui Province, China; ${ }^{3}$ Department of Gastroenterology, The First Affiliated Hospital of Zhejiang Chinese Medical University, Hangzhou, Zhejiang Province, China; ${ }^{4}$ Department of Gastroenterology, The First Affiliated Hospital of Fujian Medical University, Fuzhou, Fujian Province, China; ${ }^{5}$ Department of Gastroenterology, The First Affiliated Hospital, Medical College of Zhejiang University, Hangzhou, Zhejiang Province, China

\subsection{6/gutjnl-2018-IDDFabstracts. 164}

Background Inflammatory bowel disease (IBD) may impair patients' health-related quality of life (HRQOL) and impose burdens on caregivers. We aim to survey HRQOL including demographic characteristics of IBD patients and to evaluate the diseaserelated perception of their caregivers in East China.

Methods Patients above 18 years old with established Crohn's disease (CD) or ulcerative colitis (UC) from 5 medical centres in East China between December 2016 and July 2017 were enrolled. Patients' detailed demographic and clinical information were documented. Demographic data of the investigated regions were extracted from China Population and Employment Statistics Yearbook 2016 for comparison. Patients HRQOL was assessed by short IBD questionnaire (SIBDQ) and patient-reported $0-10$ score (IBD-10). The major caregiver of each patient was surveyed by IBD-10 to estimate patient's condition from caregiver's viewpoint.

Results A total of 601 IBD patients were included in this study. The proportion of post-secondary school education $(51.4 \%$ vs $28.1 \%, \mathrm{p}<0.0001)$ and the unemployment rate $(31.8 \%$ vs $2.9 \%-4.0 \%, \mathrm{p}<0.0001)$ was significantly higher in IBD cohort than in general population. Patients in active phase had remarkably lower SIBDQ and IBD-10 scores than those in remission, meanwhile, the scores declined along with the illness severity (table 1). Long disease duration, illness activity, unemployment and corticosteroid treatment were risk factors for poor HRQOL while high BMI provided a
A

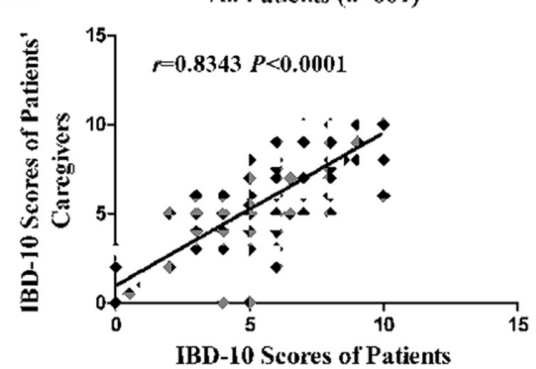

B

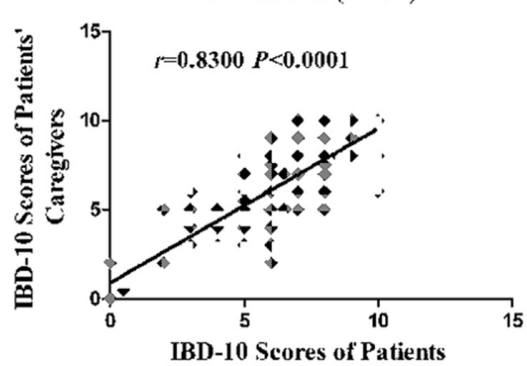

C

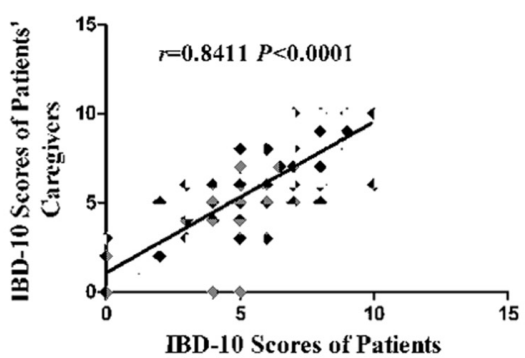

Abstract IDDF2018-ABS-0223 Figure 1 Correlation between ibd 10 scores of ibd patients and their caregivers 
protective effect. There was a strong positive correlation between IBD-10 scores of patients and their caregivers (figure $1)$.

\begin{tabular}{|c|c|c|c|c|c|}
\hline $\begin{array}{l}\text { Scores: median } \\
\text { (IQR) }\end{array}$ & Remission & Mild & Moderate & Severe & $P$-value \\
\hline CD: SIBDQ & $57(51-64)$ & $\begin{array}{c}53(44- \\
60)\end{array}$ & $\begin{array}{c}41(34- \\
47)\end{array}$ & $\begin{array}{c}32(24- \\
50)\end{array}$ & $<0.0001$ \\
\hline CD: IBD-10 & $8(6-8)$ & 7 (5-8) & $6(5-7)$ & $4(3-7)$ & $<0.0001$ \\
\hline UC: SIBDQ & $\begin{array}{c}58(49.5- \\
64)\end{array}$ & $\begin{array}{c}49(40- \\
55)\end{array}$ & $\begin{array}{c}46(34- \\
54)\end{array}$ & $\begin{array}{c}26(22- \\
32)\end{array}$ & $<0.0001$ \\
\hline UC: IBD-10 & $7.5(6-8)$ & $6.5(5-8)$ & $6(5-8)$ & $4(2-5)$ & 0.005 \\
\hline
\end{tabular}

Conclusions Higher educational attainment and unemployment rate exist among IBD patients in East China. Patients HRQOL declines and correlates with multiple negative factors. Caregivers may form similar disease-related perception just as patients do.

\section{IDDF2018-ABS-0224 EVALUATING STRATIFICATION PERFORMANCE OF HIGH-RISK FACTORS QUESTIONNAIRE (HRFQ) FOR COLORECTAL CANCER AND ADVANCED COLORECTAL NEOPLASIA IN CHINESE ASYMPTOMATIC SUBJECTS}

${ }^{1}$ Liwen Huang*, ${ }^{2}$ Shanjuan Wang, ${ }^{3}$ Ping Chen, ${ }^{3}$ Xiaogin Yuan, ${ }^{4}$ Wei Huang, ${ }^{5}$ Ming $\mathrm{Hu}$ ${ }^{6}$ Tiansheng Huang, ${ }^{7}$ Xiaogang Li, ${ }^{8}$ Yinbing Tan, ${ }^{9}$ Martin Wong. ${ }^{1}$ Chinese University of Hong Kong, Hong Kong: ${ }^{2}$ Department of Gastroenterology, Hospital of Jiading District, Shanghai, China; ${ }^{3}$ Ruijin Hospital North, Shanghai Jiaotong University, Shanghai, China; ${ }^{4}$ Department of Gastroenterology, Li Qun Hospital, Shanghai, China; ${ }^{5}$ Department of Gastroenterology, Nan Xiang Hospital, Shanghai, China; ${ }^{6}$ Department of Gastroenterology, Traditional Chinese Medicine Hospital of Jiading District, Shanghai, China; 'Department of Gastroenterology, Dachang Hospital, Shanghai, China; ${ }^{8}$ Department of Gastroenterology, Huaqiao Hospital, Kunshan, Jiangsu, China; ${ }^{9}$ Institute of Digestive Disease, Faculty of Medicine, Chinese University of Hong Kong, Hong Kong

\subsection{6/gutjnl-2018-IDDFabstracts.165}

Background High-Risk Factors Questionnaire (HRFQ) has been widely applied in Chinese organised colorectal cancer (CRC) screening programmes since the 1990s. It served as a screening test to identify the possibly missing high-risk population whose faecal occult blood test result was negative. Nevertheless, its performance to stratify CRC and advanced colorectal neoplasia (ACN) have not been adequately examined. There is no comparison between its discriminatory capability and that of other risk prediction models commonly adopted in China, such as the Asia Pacific Colorectal Screening (APCS).

Methods A prospective, multi-centre colonoscopy study was held in 7 endoscopic units across North Shanghai, China from 2016 to 2017. Average-risk subjects were recruited when they received screening colonoscopy. Information required by HRFQ, including family history of CRC in first degree relatives, personal history of any malignancy, past history of colorectal polyps, and other required variables were collected by self-reported questionnaires. Experienced colonoscopists performed all the colonoscopy, while pathologists who were blinded to the survey findings, reported the histology based on standard diagnostic criteria. The sensitivity, specificity, positive/negative predictive value(PPV/NPV), and the C-statistics were calculated to evaluate the discriminatory value of HRFQ for CRC and ACN. We also examined the above parameters when APCS scoring system was used, using a score of 4-7 points as 'high-risk'.

Results Among all 2813 enrolled individuals (mean age 58.7 \pm 8.4 years, male 52.0\%), 142 cases $(5.0 \%)$ had ACN and 29 patients $(1.0 \%)$ had invasive cancers. The proportion of highrisk population identified by HRFQ and APCS were $46.9 \%$ $(n=1,318)$ and $31.8 \%(n=894)$, respectively. For CRC the difference between HRFQ and APCS was not significant in Cstatistics. However, for ACN the performance of APCS with respect to sensitivity, specificity, PPV, NPV and C-statistics, were significantly superior to that of HRFQ (table 1).

Conclusions These findings implied that the use of HRFQ might need to be re-examined to attain a satisfactory predictive capability. Due to the current limited CRC case number, we recommend future studies in other population groups to enhance its generalizability.

Abstract IDDF2018-ABS-0224 Table 1 Predictive performance of HRFQ and APCS for CRC, ACN $(95 \% \mathrm{Cl})(\mathrm{n}=2,813)$

\begin{tabular}{lcccc}
\hline & CRC $(\mathrm{n}=29)$ & \multicolumn{3}{c}{ ACN(n=142) } \\
\hline & HRFQ & APCS & HRFQ & APCS \\
Sensitivity(\%) & $48.3(47.7,48.9)$ & $58.6(58.0,59.2)$ & $48.6(48.3,48.9)$ & $51.4(51.1,51.8)$ \\
Specificity(\%) & $53.2(53.1,53.2)$ & $68.5(68.4,68.6)$ & $53.2(53.2,53.3)$ & $69.3(69.2,69.3)$ \\
PPV(\%) & $1.1(1.0,1.1)$ & $1.9(1.9,1.9)$ & $5.2(5.2,5.3)$ & $8.2(8.1,8.2)$ \\
NPV(\%) & $99.0(99.0,99.0)$ & $99.4(99.4,99.4)$ & $95.1(95.1,95.2)$ & $96.4(96.4,96.4)$ \\
C-statistics & $0.51(0.40,0.61)$ & $0.64(0.53,0.74)$ & $0.51(0.46,0.56)$ & $0.65(0.60,0.69)$
\end{tabular}

HEFR: High Risk Factors Questionnaire; APCS: Asia Pacific Colorectal Screening; CRC: colorectal cancer, ACN: advanced colorectal neoplasia; PPV: positive predictive value; NPV: neg ative predictive value; $\mathrm{Cl}$ : confidence interval.

\section{IDDF2018-ABS-0225 FAECAL MICROBIOTA TRANSPLANTATION BY CAPSULES FOR ACTIVE UNCREATIVE COLITIS: A RANDOMISED TRIAL}

${ }^{1}$ Qiongyun Chen* ${ }^{1}$ Yanyun Fan, ${ }^{2}$ Bangzhou Zhang, ${ }^{1}$ Qingwen Huang, ${ }^{1}$ Chuanxing Xiao, ${ }^{1}$ Hongzhi Xu, ${ }^{1}$ Jianlin Ren, ${ }^{2}$ Xiang Zhang. ${ }^{1}$ Zhongshan Hospital Department of Gastroenterology, Xiamen University, Xiamen, China; ${ }^{2}$ Institute for Microbial Ecology, Medical College of Xiamen University, Xiamen, China

\subsection{6/gutjnl-2018-IDDFabstracts. 166}

Background Faecal microbiota transplantation (FMT) is a novel form of therapeutic manipulation through gastroscopy, colonoscopy, naso-intestinal tube, et al, which brings pain and inconvenience to the patients. We aim to establish an easy way of FMT in active ulcerative colitis.

Methods We conducted a randomised, double-blind trial in Zhongshan Hospital Affiliated to Xiamen University, Xiamen, China. We randomised allocated patients with active ulcerative colitis accompanied by Mayo score 5-9 to FMT, followed by capsules 3 days one week. One donor's faecal microbiota was made into one capsule, and faecal microbiota transplantation 